\title{
Dada, Surrealism, and Romania: A Bibliography Timothy Shipe
}

This selective bibliography of Dada, surrealism, and Romania is intended primarily for Anglophone and Francophone readers. Primary texts in Romanian are included; but secondary literature is generally limited to books in English and French, except where there is a dearth of material on a particular writer or artist. The focus is on monographic publications, articles being included only very selectively.

The reviews associated with Dada and surrealism in Romania are listed chronologically by initial year of publication. Similarly, under each author, primary literature is listed by year of first publication. For prolific authors and those whose Dada or surrealist connections diminished later in life, the listing of primary literature is more selective after 1950. Secondary literature is listed alphabetically by author or editor, and exhibition catalogs are listed chronologically.

Except where noted, all citations are for print publications.

\section{Reviews Associated with Romanian Dada and Surrealism}

Simbolul. Ed. Tristan Tzara, Ion Vinea, and Marcel Janco [as S. Samyro, I. Iovanaki, and Marcel Iancu]. 4 numbers (1912).

Chemarea [first series]. Ed. Ion Vinea. 2 numbers (1915).

Contimporanul. Ed. Ion Vinea. 102 numbers (1922-32).

75 HP. Ed. Victor Brauner and Ilarie Voronca. 1 number (Oct. 1924). Facsim. rpt.:

Paris: Jean-Michel Place, 1993.

Punct. Ed. Scarlat Callimachi. 16 numbers (1924-25). Facsim. rpt.: Bucharest: ICARE, 2003.

Integral. Ed. Ilarie Voronca. 15 numbers (1924-25).

Urmuz. Ed. Geo Bogza. 5 numbers (1928).

Unu. Ed. Saşa Pană. 50 numbers (1928-32). Title in most issues printed all lowercase: $u n u$.

Alge. Ed. Aureliu Baranga. Series 1: 8 numbers (1930); Series 2: 3 numbers (1933).

Pula. Ed. Gherasim Luca, Paul Păun, S. [Jules] Perahim, and Aureliu Baranga. 1 number (Oct. 1931). 
Muci. Ed. Aureliu Baranga, S. [Jules] Perahim, Gherasim Luca, Paul Păun, and Sesto Pals. 1 number (7 Feb. 1932).

Liceu. Ed. Virgil Teodorescu. 2 numbers (1932).

Viaţa imediată. Ed. Geo Bogza. 1 number (Dec. 1933).

\section{General Monographs}

Benson, Timothy O., and Éva Forgács, eds. Between Worlds: A Sourcebook of Central European Avant-gardes, 1910-1930. Los Angeles: Los Angeles County Museum of Art; Cambridge: MIT P, 2002.

Cârneci, Magda. București anii 1920-1940: Intre avangardă și modernism (Bucharest in the 1920s-1940s: Between Avant-garde and Modernism). Bucharest: Simetria; Uniunea Arhitecților din România, 1994.

Cernat, Paul. Avangarda românească şi complexul periferiei. Bucharest: Cartea Românească, 2007. Critică \& istorie literară.

Colonaş, Florin. Revizitând avangarda. Ed. Petrişor Militaru. Craiova: Aius, 2013. Avangardă \& transdisciplinaritate.

Crohmălniceanu, Ovid S. Evreii în mişcarea de avangardă românească. Bucharest: Hasefer, 2001.

David, Emilia. Futurismo, dadaismo e avanguardia romena: Contaminazioni fra culture europee (1909-1930). Torino: Harmattan Italia, 2006. Indagini e prospettive 15.

Gibus, Aurélia. "Parcourir l'impossible: La Construction du surréalisme en Roumanie." 2 vols. MA thesis. Université Paris Ouest, 2007.

Ilk, Michael. Brancusi, Tzara und die rumänische Avantgarde. Bochum: Museum Bochum, 1997.

Manucu, Nicole. De Tristan Tzara à Ghérasim Luca: Impulsions des modernités roumaines au sein de l'avant-garde européenne. Paris: Champion, 2014. Bibliothèque de littérature générale et comparée 126.

Militaru, Petrişor. Ştiinţa modernă, muza neştiută a suprarealiştilor. Bucharest: Curtea Veche, 2012. Ştiință, spiritualitate, societate.

Morar, Ovidiu. Avangardismul românesc. Bucharest: Ideea Europeană, 2005. Colecția Istorie, teorie și critică literară.

—. Avatarurile suprarealismului românesc. Bucharest: Univers, 2003.

Pop, Ion. Avangarda în literatura română. Bucharest: Minerva, 1990. Momente și sinteze.

—. Din avangardă spre ariergardă. Bucharest: Vinea, 2010.

- Introducere în avangarda literară românească. Bucharest: Institutul Cultural Român, 2007.

ed. La Réhabilitation du rêve: Une Anthologie de l'avant-garde roumaine. Ed. Ion Pop. Paris: Nadeau; Bucharest: Institutul Cultural Român, 2006.

Răileanu, Petre, ed. The Romanian Avant-garde. Spec. issue of Plural 3 (1999).

Sandqvist, Tom. Dada East: The Romanians of Cabaret Voltaire. Cambridge: MIT P, 2006. 
Stern, Radu, and Edward van Voolen, eds. Van Dada tot surrealisme: Joodse avantgarde kunstenaars uit Roemenië, 1910-1938 (From Dada to Surrealism: Jewish Avantgarde Artists from Romania, 1910-1938). Amsterdam: Joods Historisch Museum, 2011.

Le Surréalisme roumain. Paris: Paris-Méditerranée, 2004. Seine et Danube 3.

Tănase, Stelian. Avangarda românească în arhivele siguranţei. Bucharest: Polirom, 2008. Istorii subterane.

Vanci, Marina. “Concept de modernisme et d'avant-garde dans l'art roumain entre les deux guerres." Diss. (Thèse de 3e cycle). Université Paris 1, 1972.

Velescu, Cristian-Robert. Avant-gardes et modernités: Brancusi, Duchamp, Brauner, Tzara E comp. Bucharest: Institutul Cultural Român, 2013. Argumente.

Yaari, Monique, ed. "Infra-noir" un et multiple: Un Groupe surréaliste entre Bucarest et Paris, 1945-1947. Oxford: P. Lang, 2014.

\section{General Articles}

Eburne, Jonathan P. "“Comme une érosion unique': Les Provocations d'Infra-noir." Yaari, Monique, ed. "Infra-noir," un et multiple 33-35.

Ierunca, Virgil. "Roumanie." Dictionnaire général du surréalisme et de ses environs. Ed. Adam Biro and René Passeron. Fribourg: Office du livre, 1982. 369-70.

Impey, Michael H. "Before and After Tzara: Romanian Contributions to Dada. The Eastern Dada Orbit: Russia, Georgia, Ukraine, Central Europe and Japan. Ed. Stephen C. Foster. New York: G.K. Hall; London: Prentice Hall, 1998. 126-136. Lecherbonnier, Bernard. "Le S.O.S. roumain: Un Radical Rappel à l'ordre." La Chair du verbe: Histoire et poétique des surréalismes de langue française. Paris: Publisud, 1992. 57-61. Collection Littératures.

Pop, Ion. “Dada en Roumanie: Échos et prémisses." Dada Circuit Total. Ed. Henri Béhar and Catherine Dufour. Lausanne: Age d'Homme, 2005. 82-89. Les Dossiers H.

—. "Repères pour une histoire du surréalisme roumain." Mélusine 11 (1990): 143-55.

Rind, Nina. "Free of History - Mal d'ombre: Romania and Surrealism." Conscious Hallucinations: Filmic Surrealism. Ed. Svetlana Svyatskaya. Frankfurt am Main: Deutsches Filmmuseum; Munich: Belleville, 2014. 140-45.

Sarré, Jean-Luc. "En Roumanie." La Planète affolée: Surréalisme, dispersion et influences, 1938-1947. Marseille: Musées de Marseille; Paris: Flammarion, 1986. 256-61.

Vanci-Perahim, Marina. “Le Surréalisme en Roumanie: Un peu, beaucoup, passionnément." Opus international 123-24 (April-May 1991): 136-40.

Yaari, Monique. "Le Groupe surréaliste de Bucarest entre Paris et Bruxelles, 19451947: Une page d'histoire." Synergies Canada 3 (2011). Web.

<http://synergies.lib.uoguelph.ca/article/view/1468/2321>. 
. "The Surrealist Group of Bucharest: Collective Works, 1945-1947." Paris-

Bucharest, Bucharest-Paris: Francophone Writers from Romania. Ed. Anne Quinney. Amsterdam: Rodopi, 2012. Faux Titre 367. 95-136.

\section{Blecher}

Born Max L. Blecher, 1909, Botoşani, Romania; died 1938, Roman, Romania.

\section{PRIMARY}

Corp transparent. Bucharest: Bibliofilă, 1934.

Intâmplări în irealitatea imediată. Bucharest: Vremea, 1936. Trans. as Occurrence in the Immediate Unreality. Trans. Alistair Ian Blyth. Plymouth: U of Plymouth P, 2009. 20 Romanian Writers Ser. Trans. as Adventures in Immediate Irreality. Trans. Michael Henry Heim. New York: New Directions, 2015.

Inimi cicatrizate. Bucharest: Universala, 1937. Trans. as Scarred Hearts. Trans. Henry Howard. London: Old Street, 2008.

Viziuna luminată; Corp transparent, proze, publicistică, archivă. Ed. Saşa Pană. Bucharest: Cartea românească, 1971.

Opere complete. Ed. Constantin M. Popa and Nicolae Țone. Craiova: Aius; Bucharest: Vinea, 1999.

\section{SECONDARY}

Brăvescu, Ada. M. Blecher: Un caz de receptare problematic şi spectaculos. Bucharest: Tracus Arte, 2011.

Glăvan, Gabriela. "Mapping the Unreal: Max Blecher in the Shadow of the Avantgarde." Arcadia 49.1 (2014): 1-20.

Glodeanu, Gheorghe. Max Blecher şi noua estetică a romanului românesc interbelic. Cluj-Napoca: Limes, 2005.

Ifrim, Nicoleta. "Ego Games in Blecher's Mirror-Writing." Communication interculturelle et littérature 2 (Aug. 2008): 45-48.

Mironescu, Doris. Viaţa lui M. Blecher: Impotriva biografiei. Iaşi: Timpul, 2011.

\section{Geo Bogza}

Born 1908, Blejoi, Romania; died 1993, Bucharest.

\section{PRIMARY}

Jurnal de sex: Poeme. Bucharest: Integral, 1929.

Poemul invectivă. Bucharest: Unu, 1933. Rpt.: Ed. Nicolae Tzone. Bucharest: Vinea, 2009. Avangarda.

Ioana Maria: Şaptesprezece poeme. Bucharest: Azi, 1937.

Ţări de piatră, de foc şi de pământ. Bucharest: Fundația Regele Carol II, 1939. Scriitorii români contemporani. Rpt.: Pref. Paul Cernat. Bucharest: Jurnalul Naţional, 2011. Biblioteca pentru toți 137. Trans. as Land of Stone, the Land of the Motzi. Bucharest: “Book" Pub. House, 1954. 
Cântec de revoltă, de dragoste şi moarte. Bucharest: Fundaţia Regele Mihai 1, 1945.

Cartea Oltului. Bucharest: Fundaţia Regele Mihai I., 1945. Rpt.: Bucharest: Albatros, 1979. Colecția Lyceum 227. Rpt.: Bucharest: Jurnalul Naţional, 2012. Biblioteca pentru toți ; 146.

Oameni şi cărbuni în Valea Jiului. Bucharest: Ed. de Stat, 1947.

Sfârşitul lui Iacob Onisia. Bucharest: Ed. Pentru Literatură şi Artă, 1949.

Anii impotrivirii: Reportaje, pamflete, articole, 1934-1939. Bucharest: Ed. Tineretului, 1953. Trans. as Years of Darkness. Trans. Lazar Marinescu. Bucharest: "Book" Pub. House, 1955.

Orion. Bucharest: Minerva, 1978.

Jurnal de copilărie şi adolescență. Bucharest: Cartea Românească, 1987.

Eu sunt Ținta: 18 iulie 1992, Snagov-14 septembrie 1993, Spitalus Elias. Bucharest: Du Style, 1996. (Interviews with Diana Turconi.)

Poemul invectivă şi alte poeme. Pref. Paul Cernat. Bucharest: Jurnalul Naţional, 2010.

Biblioteca pentru toți 89. (Collected poems.)

\section{SECONDARY}

Crohmălniceanu, Ov. S. "Supreme Simplicity." Rumanian Review 33.1 (1979): 7-9. Ignatović, Srba. "Skeptical Optimism." Rumanian Review 41.4 (1987): 111-12.

Lascu, Mădălina. Epistolar avangardist: Corespondență primită de Geo Bogza de la Stephan Roll, Saşa Pană, Mary-Ange Pană, Victor Brauner, Ilarie Voronca, Colomba Voronca, grupul "Alge." Bucharest: Tracus Arte, 2012. Biblioteca avangardei.

Petrov, Aleksandar. “Bogza's Key.” Rumanian Review 41.4 (1987): 109-11.

Scărlătescu, Doru. Geo Bogza. Bucharest: Minerva, 1983.

Steinhardt, Nicolae. Geo Bogza: Un poet al efectelor, exaltării, grandiosului, solemnităţii, exuberanţei şi patetismului. Bucharest: Albatros, 1982. Rpt.: Ed. George Ardeleanu. Rohia: Mănăstirea Rohia; Iaşi: Polirom, 2011.

Tănăsescu, Antoaneta. Geo Bogza. Bucharest: Eminescu, 1976. Biblioteca critică.

\section{Victor Brauner}

Born 1903, Piatra Neamț, Romania; died 1966, Paris.

\section{PRIMARY}

Victor Brauner: Écrits et correspondances 1938-1948. Ed. Camille Morando and Sylvie Patry. Paris: Centre Georges Pompidou, 2005.

\section{SECONDARY}

Alexandrian, Sarane. "Victor Brauner." L'CEil, 101 (May 1963): 32-39, 68.

- Victor Brauner. Paris: Oxus, 2004. Les Étrangers de Paris: Les Roumains de Paris.

- Victor Brauner l'illuminateur. Paris: Cahiers d'art, 1954.

Flahutez, Fabrice. "La Bibliothèque de Victor Brauner et la bibliothèque d'André Breton: Surprises et limites du comparatisme." Les Bibliothèques d'artistes: XXe- 
XXIe siècles. Ed. Françoise Levaillant, Dario Gamboni, and Jean-Roch Bouiller. Paris: Presses de 1'Université Paris-Sorbonne, 2010. 107-27.

- . "Figurer l'inconscient dans le dur: Les Totems de Victor Brauner et d'Anton Prinner." Hypnos: Images et inconscients en Europe (1900-1949). Lille: Musée d'art moderne Lille métropole, 2009. 264-75.

Hoctin, Luce. “Propos sur Victor Brauner.” Quadrum 15 (1963): 55-70. English summary on p. 179.

Jouffroy, Alain. "Suprématie poétique de Victor Brauner." Cahiers d'art 26 (1951): 162-70.

- Victor Brauner. Paris: Fall, 1996.

Morando, Camille. “'Térapeutique [sic] de démoralisation ou contre inspiration' chez Victor Brauner: L'Invention d'une écriture dictée par la privation et l'absence." Arts drogués: Expériences psychotropiques et création artistique. Ed. Fabrice Flahutez and Miguel Egaña. Nanterre: Presses universitaires de Paris Ouest, 2013. 149-60.

Nicolae, Emil. Patimile după Victor Brauner. Bucharest: Hasefer, 2006.

- Victor Brauner: La izvoarele operei. Bucharest: Hasefer, 2004.

- Victor Brauner şi însoțitorii: Incursiuni în avangardă. Bucharest: Hasefer, 2013.

Semin, Didier. Victor Brauner. Paris: Réunion des musées nationaux; Filipacchi, 1990.

Vallier, Dora. "Petite introduction à la peinture de Brauner." XXe Siècle N.S. 25.22 (Christmas 1963): 29-38.

Vanci-Perahim, Marina. “Le Lisible et le visible: Victor Brauner de la 'picto-poésie' à la 'hiéroglyphisation des sentiments.'” Mélusine 12 (1991): 63-70.

Velescu, Cristian. Victor Brauner d'après Duchamp, sau, Drumul pictorului către un suprarealism "bine temperat." Bucharest: Institutul Cultural Român, 2007.

\section{EXHIBITION CATALOGUES}

Musée national d'art moderne. Victor Brauner. By Dominique Bozo. Paris: Réunion des musées nationaux, 1972. 2 June-25 Sept. 1972.

Victor Brauner. Paris: Réunion des musées nationaux; Filipacchi, 1990. 1990.

Musée des Sables d'Olonne. Les Victor Brauner de la collection de l'Abbaye SainteCroix. Ed. Didier Ottinger. Les Sables d'Olonne: Musée des Sables d'Olonne, 1991. Feb.-April 1991.

Musée d'art moderne Saint-Etienne and Musée d'Unterlinden. Victor Brauner. Saint-Etienne: Musée d'art moderne; Colmar: Musée d'Unterlinden, 1992. 1992.

Galleria Credito valtellinese. Victor Brauner, 1903-1966. Ed. Dominique Stella. Milan: Mazzotta, 1995. May-June 1995.

Centre Georges Pompidou. Victor Brauner dans les collections du MNAM-CCI. By Didier Semin. Paris: Centre Georges Pompidou, 1996. Collections du Musée national d'art moderne. 24 Jan.-6 May 1996. 
Fondation de L'Hermitage. Victor Brauner, ou, L'enchantement surréaliste. Lausanne: Fondation de L'Hermitage, 1999. 9 July-10 Oct. 1999.

Menil Collection. Victor Brauner: Surrealist hieroglyphs. Houston: Menil Collection; Ostfildern-Ruit: Hatje Cantz, 2001. 12 Oct. 2001-6 Jan. 2002.

Musée des beaux-arts de Brest. Victor Brauner: Un monde magique. Brest: Musée de Brest, 2009. 8 July-31 Oct. 2009.

Galerie Schoffel-Valluet and Galerie Samy Kinge. Dialogue des mondes: Victor Brauner et les arts primitifs. Paris: Galerie Schoffel-Valluet, Galerie Samy Kinge, 2010. 21 Oct.-21 Dec. 2010.

\section{Benjamin Fondane}

Born Benjamin Wechler, 1894, Iaşi; died 1944, Auschwitz. Signed "B. Fundoianu" for works in Romanian, "Benjamin Fondane" for works in French.

\section{PRIMARY}

Tăgăduința lui Petru: Literatură biblică: Cu o lămurire despre simbolism. Iași: Chemarea, 1918. Rpt. in Imagini și cărți din franța (1980).

Imagini și cărți din Franța. Bucharest: Socec, 1921. Rpt.: Ed. Vasile Teodorescu. Bucharest: Minerva, 1980. (Includes most of the author's Romanian prose texts and selected French texts in translation.) Bucharest: Institutul Cultural Român, 2006. French trans. as Images et Livres de France. Trans. Odile Serre. Paris : ParisMéditerranée, 2002.

Trois scenarii: Ciné-poèmes de Benjamin Fondane. Brussels: Documents internationaux de l'esprit nouveau, 1928.

Priveliști: Poeme, 1917-1923. Bucharest: Cultura națională, 1930.

Ulysse. Brussels: Journal des poètes, 1933. Les Cahiers du Journal des poètes.

Rimbaud le voyou. Paris: Denoël et Steele, 1933. Rev. as Rimbaud le voyou et l'expérience poétique. Ed. Michel Carassou. Paris: Plasma, 1979. Rpt.: Paris: Non lieu, 2010.

La Conscience malheureuse. Paris: Denoël et Steele, 1936. Rev. ed. Ed. Olivier SalazarFerrer. Lagrasse: Verdier; Paris: Non lieu, 2013.

Titanic: Poèmes. Brussels: Journal des poètes, 1937. Les Cahiers du Journal des poètes.

Faux traité d'esthétique: Essai sur la crise de réalité. Paris: Denoël, 1938. Rpt.: Ed. Catherine Thieck. Paris: Plasma, 1980. Rpt.: Ed. Ann Van Sevenant. Paris: ParisMéditerranée, 1998.

Baudelaire et l'expérience du gouffre. Paris : Seghers, 1947. Rpt. Paris: Seghers, 1972. Rpt.: Paris: Complexe, 1994.

Poezii. Ed. Virgil Teodorescu. Bucharest: Ed. Pentru Literatură, 1965. (Collected Romanian poems, with trans. by Teodorescu of French poems.)

Le Mal des fantômes, précédé de Paysages. Paris: Plasma, 1980. Collected French poems. Rev. ed.: Ed. Patrice Beray and Michel Carassou. Lagrasse: Verdier, 2006. 
Écrits pour le cinéma: Le Muet et le parlant. Ed. Michel Carassou, Olivier SalazarFerrer, and Ramona Fotiade. Paris: Plasma, 1984. Nouv. éd. Paris: Non lieu; Lagrasse: Verdier, 2007.

L'Écrivain devant la révolution: Discours non prononcé au Congrès international des écrivains de Paris (1935). Paris: Paris-Méditerranée, 1997.

Opere. Ed. Paul Daniel, George Zarafu, and Mircea Martin. 2 vols. to date. Bucharest: Art, 2011- . Seria Fundoianu-Fondane. (Complete edition of Fondane's Romanian and French works; French texts in Romanian translation only.)

Théâtre complet. Ed. Eric Freedman. Paris: Non lieu, 2012.

Benjamin Fondane entre littérature et philosophie. Ed. Monique Jutrin. Paris: Parole et Silence, 2015.

\section{SECONDARY}

Benjamin Fondane. Spec. issue of Europe 827 (March 1998).

Beray, Patrice. Benjamin Fondane, au temps du poème: Essai. Toulouse: Les Amis de l'éther vague; Lagrasse: Verdier, 2006.

Cahiers Benjamin Fondane. 18 vols. to date. Kfar-Saba, Israel: Société d'études Benjamin Fondane, 1997-

Carassou, Michel, ed. Benjamin Fondane. Spec. issue of Non lieu 2-3 (1978).

Carassou, Michel. "Benjamin Fondane et la conscience honteuse du surréalisme." Mélusine 3 (1982): 181-90.

Finkenthal, Michael. Benjamin Fondane: A Poet-Philosopher Caught Between the Sunday of History and the Existential Monday. New York: P. Lang, 2013.

Finkenthal, Michael, Claire Gruson, and Roxana Sorescu, eds. B. Fundoianu Benjamin Fondane: O nouă lectură. Iaşi: Ed. Universităţii “Alexandru Ioan Cuza," 2013. (Essays in English, French, and Romanian.)

Fotiade, Ramona. Conceptions of the Absurd: From Surrealism to the Existential Thought of Chestov and Fondane. Oxford: Legenda, 2001.

Fotiade, Ramona, ed. The Tragic Discourse: Shestov and Fondane's Existential Thought. Oxford: P. Lang, 2006. European Connections 26.

Freedman, Eric. Bibliographie des auvres publiées de Benjamin Fondane 1912-2008. Paris: Non lieu; Société d'études Benjamin Fondane, 2009.

Grün, Ecaterina. La Route chez Tristan Tzara, Benjamin Fondane et Ilarie Voronca. Cordes-sur-Ciel: Surtis, 2006. Collection "Art, artistes."

Hatem, Jad. Semer le messie selon Fondane poète. Brussels: Part de l'œil, 2004. Collection Fiction 6.

Hyde, John Kenneth. Benjamin Fondane: A Presentation of His Life and Works. Geneva: Droz, 1971. Histoire des idées et critique littéraire 112.

Jutrin, Monique. Avec Benjamin Fondane au-delà de l'histoire ou les carnets d'Ulysse, 1924-1944. Paris: Parole et Silence, 2001.

—. Benjamin Fondane ou le périple d'Ulysse. Paris: Nizet, 1989. 
Jutrin, Monique, ed. Rencontres autour de Benjamin Fondane, poète et philosophe : Actes du Colloque de Royaumont, 24, 25 et 26 avril 1998. Paris : Parole et silence, 2002

Jutrin, Monique. "The Trans-cultural Journey of Benjamin Fondane." ParisBucharest, Bucharest-Paris: Francophone Writers from Romania. Ed. Anne Quinney. Amsterdam: Rodopi, 2012. Faux Titre 367. 137-69.

Kluback, William. Benjamin Fondane: A Poet in Exile. New York: P. Lang, 1996. The Literature and Poetry of Exile 1.

Lucescu Boutcher, Arta. Rediscovering Benjamin Fondane. New York: P. Lang, 2003. Currents in Comparative Romance Languages and Literatures 91.

Meffre, Liliane, and Olivier Salazar-Ferrer, eds. Carl Einstein et Benjamin Fondane: Avant-gardes et émigration dans le Paris des années 1920-1930. Brussels: P. Lang, 2008. Comparatisme et société 6 .

Mounic, Anne, ed. Benjamin Fondane et Claude Vigée: Le Questionnement des origines. Paris: Champion, 2014. Colloques, congrès et conférences sur la littérature comparée 22.

Pop, Ion. "Benjamin Fondane dans les revues roumaines d'avant-garde." Anuar de linguistică şi istorie literară 34.8 (1994): 207-14.

Răileanu, Petre, and Michel Carassou, eds. Fundoianu/Fondane et l'avant-garde. Bucharest: Fondation culturelle roumaine; Paris: Paris-Méditerranée, 1999.

Salazar-Ferrer, Olivier. Benjamin Fondane. Paris: Oxus, 2004. Les Étrangers de Paris. Les Roumains de Paris.

Benjamin Fondane et la révolte existentielle: Essai. Brussels: Corlevour, 2008.

\section{Gherasim Luca}

Born Salman Locker, 1913, Bucharest; died 1994, Paris. Changed his surname to Gherasim Luca. Signed work "Ghérasim Luca" (with and without accent) after emigration to France; considered this a compound surname, without any given name.

PRIMARY

Roman de dragoste. Bucharest: Colecţia Alge, 1933.

Fata Morgana. Craiova: Ateliere Ramuri, 1937.

Inventatorul iubirii, urmat de Parcurg imposibilul şi de Moartea moartă. Bucharest:

Negaţia Negației, 1945. French trans. as L'inventeur de l'amour, suivi de La Mort morte. Trans. Ghérasim Luca. Paris: José Corti, 1994. Trans. as The Inventor of Love $\mathcal{E}$ Other Writings. Trans. Julian and Laura Semilian. Boston: Black Widow $\mathrm{P}, 2009$.

Le Vampire passif. Paris [i.e. Bucharest]: Éd. de l'Oubli, 1945. Rev. ed. Paris: José Corti, 2001. Trans. as The Passive Vampire. Trans. Krzysztof Fijałkowski. Prague: Twisted Spoon P, 2008.

Un Lup văzut printr'o lupă. Bucharest: Negația Negaţiei, 1945. Trans. as Un loup à travers une loupe. Trans. Ghérasim Luca. Paris: J. Corti, 1998. Domaine français. 
Les Orgies des Quanta: Trente-trois cubomanies non-oedipiennes. Bucharest: Surréalisme, 1946.

Amphitrite: Mouvements sur-thaumaturgiques et non-oedipiennes. Bucharest: Imp. Socec, 1947. Infra-noir. Facsim. rpt. in Infra-noir rpt. (1996) and in Yaari, ed., "Infra-noir" un et multiple 302-09.

Le Secret du vide et du plein. Bucharest: Imp. Socec, 1947. Infra-noir. Facsim. rpt. in Infra-noir rpt. (1996) and in Yaari, ed., "Infra-noir" un et multiple 310-17.

Niciodată destul. Bucharest: Negația Negației, 1947.

Héros-Limite. Paris: Soleil Noir, 1953. Rpt.: Héros limite; suivi de Le chant de la carpe et de Paralipomènes. Paris: Gallimard, 2001. Poésie 364.

Ce château pressenti. Paris: Méconnaissance, 1958.

L'Extrême-occidentale: Sept rituels. Lausanne: Meyer, 1961.

La Lettre. Paris: n.p., 1960.

Crier taire sourire fou: Éclairage du double réglé par Gherasim Luca, Gilles Ehrmann et Micheline Catti. Paris: n.p., 1961.

Sept slogans ontophoniques. Paris: Robert Altmann, 1964. Brunidor Portfolio 4. Rpt.

(with other works): Paris: José Corti, 2008.

Sisyphe géomètre. Geneva: Claude Givaudan, 1966. (Print/Sculpture, with Piotr Kowalski.)

Apostroph' apocalypse. Milan: Upiglio, 1967.

Droit de regard sur les idées. Paris: Brunidor, 1967.

Le Chant de la carpe. Paris: Soleil Noir, 1973. Rpt.: Paris: José Corti, 1986; also rpt. with Héros-limite (2001).

Paralipomènes. Paris: Soleil Noir, 1976.

Théatre de bouche. Paris: Criapl'e, 1984. Rpt.: Paris: José Corti, 1987.

La Proie s'ombre. Paris: José Corti, 1991. Trans. as Self-Shadowing Prey. Trans. Mary Ann Caws. New York: Contra Mundum P, 2013.

La voici la voie silanxieuse. Paris: José Corti, 1997.

Levée d'écrou. Paris: José Corti, 2003.

\section{SECONDARY}

Alexandrian, Sarane. L'Évolution de Ghérasim Luca à Paris (Evoluţia lui Ghérasim Luca la Paris). Ed. and trans. Nicolae Tzone, Ioan Prigoreanu, and Marilena Munteanu. Bucharest: Vinea, 2006.

Bricianer, Serge. "Interview" [with Ghérasim Luca]. Oiseau-Tempête 4 (1998): 32.

Carlat, Dominique. Ghérasim Luca l'intempestif. Paris: José Corti, 1998.

Dossier Paul Celan Ghérasim Luca. Spec. issue of Cahier critique de poésie 17 (2008).

Fijalkowski, Krzysztof. "From Sorcery to Silence: The Objects of Gherasim Luca." Modern Language Review 88.3 (1993): 625-38.

. "Gherasim Luca: Reinvent Everything." Phosphor 3 (2011): 3-11.

. "La Poésie sans langue: Ghérasim Luca, Visual Poet." Hyperion 7.2 (2013).

Web. 6 Sept. 2016. <http://issuu.com/contramundum/docs/hyp-luca-full issue2013/13> 
Ghérasim Luca. Spec. issue of Cahiers de l'Abbaye Sainte-Croix 110 (2008).

Gibus, Aurélia. “Ghérasim Luca et les livres." 2 vols. MA thesis. Université Paris Ouest, 2006.

Graubard, Allan. Ghérasim Luca Centenary Issue (1913-1994). Spec. issue of Hyperion 7.2 (2013). Web. 3 Oct. 2015. <http://issuu.com/contramundum/docs/hyp-lucafull issue-2013/13>

Martin, Serge. "S'asseoir sans chaise avec les cubomanies et les ontophonies de Ghérasim Luca." Diérèse 46 (2009): 171-86.

Orlandi, Sibylle. "Les Cubomanies de Ghérasim Luca: Dispositif sémiotique et métalangage visuel." MA thesis. École Normale Supérieure de Lyon, Université de Lyon II, 2012.

Petreu, Marta. "Les Idées politiques de Gherasim Luca dans sa période roumaine." Synergies Roumanie 2 (2007): 57-64.

Pop, Ion. "Gherasim Luca, poète franco-roumain." Plurilinguisme et avant-gardes.

Ed. Franca Bruera and Barbara Meazzi. Brussels: P. Lang, 2011. Comparatisme et société 12. 227-39.

Răileanu, Petre. Gherasim Luca. Paris: Oxus, 2004. Les Étrangers de Paris: Les Roumains de Paris.

Toma, Iulian. Gherasim Luca ou l'intransigeante passion d'être. Paris: Champion, 2013. . “Gherasim Luca, poète d'après Auschwitz." Synergies Canada 3 (2011). Web. <http://synergies.lib.uoguelph.ca/article/view/1384/2237>.

Torlini, Yannick. Ghérasim Luca, le poète de la voix: Ontologie et érotisme. Paris: Harmattan, 2011. Approches littéraires.

Velter, André. Ghérasim Luca: Passio passionnément. Paris: Jean-Michel Place, 2001.

\section{EXHIBITION CATALOGUES}

Centre international de poésie Marseille. Ghérasim Luca: Dessins et cubomanies. Marseille: Centre international de poésie, 1991. Le Cahier du refuge 12. 28 June-31 Aug. 1991.

Espace Sculfort. Ghérasim Luca, Catti. Maubeuge: Espace Sculfort, 2000.

Abbaye Sainte-Croix, Centre international de poésie Marseille, and Centre des livres d'artistes, Saint-Yrieix-la-Perche. Ghérasim Luca. Les Sables d'Olonne: Abbaye Sainte-Croix, 2008. Cahiers de l'Abbaye Sainte Croix 110. Les Sables d'Olonne, 19 Jan.-13 April 2008; Marseille, 11 July-15 Sept. 2008; Saint-Yrieixla-Perche, Feb.-15 June 2009.

\section{Marcel Janco}

Born Marcel Hermann Iancu, 1895, Bucharest; died 1984, Tel Aviv.

PRIMARY

“Creative Dada (Schöpferischer Dada) (Dada créateur)." Dada: Monograph of a movement (Monographie einer Bewegung) (Monographie d'un mouvement). Ed. Willy Verkauf. Teufen: Niggli, 1957. 26-49. 
“Janco/Dada: An Interview with Marcel Janco." By Francis M. Naumann. Arts Magazine 57.3 (Nov. 1982): 80-86.

\section{SECONDARY}

Anghel, Doina. Marcel Iancu: Biroul de studii moderne (Bureau of modern studies). Bucharest: Simetria, 2008.

Mendelson, Marcel L. Marcel Janco. Tel Aviv: Massadah, 1962.

Seiwert, Harry. Marcel Janco: Dadaist - Zeitgenosse - wohltemperierter morgenländischer Konstruktivist. Frankfurt am Main: P. Lang, 1993. Europäishe Hochschulschriften: Reihe XXVIII, Kunstgeschichte 173.

Şerban, Geo. Intâlniri cu Marcel Iancu. Bucharest: Hasefer, 2011.

Seuphor, Michel. Marcel Janco. Amriswil: Bodensee-Verlag, 1963. Artistes de notre temps 14 .

Yoffe, A. B . Marcel Janco. Ramat-Gan: Masadah, 1982. Title also in Hebrew.

\section{EXHIBITION CATALOGUES}

Galleria Schwarz. Janco. Milan: Galleria Schwarz, 1961. Catalogo mensile 20. 21 Oct.-10 Nov. 1961.

Galerie Denise René. Marcel Janco. Paris: Galerie Denise René, 1963. Oct.-Nov. 1963.

Haifa Museum of Modern Art. Marcel Janco: Exposition rétrospective. N.p.: n.p., 1968. Traveling exhibition, spring-summer 1968. Title also in Hebrew.

Tel Aviv Museum. Marcel Janco: Exposition rétrospective. Tel Aviv: Tel Aviv Museum, 1972. June-July 1972. Title also in Hebrew.

Janco-Dada Museum. Janco-Dada Museum, Ein-Hod: Opening Exhibition. Ein-Hod: Janco-Dada Museum, 1983. Summer 1983. Title also in Hebrew.

Janco-Dada Museum. In the Struggle: Marsel Janco, Painting of the Forties. Ein-Hod: Janco-Dada Museum, 1988. Spring 1988. Title also in Hebrew.

Muzeul Național de Artă al României. Marcel Iancu în România interbelică: Arhitect, artist plastic, teoretician. Bucharest: Simetria; Uniunea Arhitecților din România; Meridiane, 1996. Exhibition “Centenar Marcel Iancu, 1895-1995,” 1996.

Antiquariat Zerfass \& Linke, Berlin, and Kunsthal Rotterdam. Marcel Janco: Das graphische Werk: Catalogue raisonné. Ed. Michael Ilk. N.p.: n.p., 2001. Berlin, 26 Oct.-14 Nov. 2001; Rotterdam, Sept.-Oct. 2002.

Colors Art Gallery. Marcel Iancu 1895-1984: Un Visionnaire de l'art moderne. Bucharest: Colors Art Gallery, 2012. 25 May-15 June 2012.

\section{Gellu Naum}

Born 1915, Bucharest; died 2001, Bucharest.

\section{PRIMARY}

Drumețul incendiar. N.p.: n.p., 1936.

Libertatea de a dormi pe o frunte. Bucharest: n.p., 1937. 
Vasco de Gama. Bucharest: Rotativa, 1940.

Culoarul somnului. Bucharest: n.p., 1944.

Medium. Bucharest: Ed. Modernă, 1945.

Teribilul interzis. Bucharest: Văcărescu, 1945. Colecția Suprarealistă.

Castelul orbilor. Bucharest: Bucovina, 1946. Colecția Suprarealistă.

Soarele calm: Poeme. Bucharest: Ed. Pentru Literatură, 1961.

Athanor: Poeme. Bucharest: Ed. Pentru Literatură, 1968.

Copacul-animal. Bucharest: Eminescu, 1971.

Tatăl meu obosit: Pohem. Bucharest: Cartea Românească, 1972. Trans. as My Tired

Father: Pohem. Trans. James Brook. Copenhagen: Green Integer, 1999. Green Integer 19.

Zenobia. Bucharest: Cartea Românească, 1985. Trans. James Brook and Sasha Vlad.

Evanston, Illinois: Northwestern UP, 1995.

Ascet la baraca de tir: Poeme. Bucharest: Ed. Fundației Culturale Române, 2000.

Calea șearpelui. Ed. Simona Popescu. Pitești: Paralela 45, 2002.

Despre interior-exterior: Gellu Naum în dialog cu Sanda Roșescu. Pitești: Paralela 45, 2003.

Despre identic şi felurit: Antologie. Ed. Simona Popescu. Iaşi: Polirom, 2004.

Biblioteca Polirom: Seria Antologii.

Opere. Ed. Simona Popescu. 3 vols. to date (4 planned). Iaşi: Polirom, 2011- .

Athanor $\mathcal{E}$ Other Poems. Trans. Margento and Martin Woodside. Philadelphia: Calypso, 2013.

\section{SECONDARY}

Athanor: Caietele Fundației "Gellu Naum." 3 vols. to date. Bucharest: Herg Benet, 2004- .

Centenar Gellu Naum. Spec. issue of Caietele avangardei 5 (2015).

Gongonea, Silviu. Gellu Naum: Aventura suprarealistă. Craiova: Aius, 2013. Avangardă \& Transdisciplinaritate.

Laville, Rémy. Gellu Naum: Poète roumain prisonnier au château des aveugles. Paris: Harmattan, 1994.

Ologu, Alina. Gellu Naum: Experimentul poetic. Constanţa: Pontica, 2007.

Orlich, Ileana Alexandra. Avantgardism, Politics, and the Limits of Interpretation in

The (Ex)centric Waste Land: Reading Gellu Naum's Zenobia. Bucharest: Paideia, 2010.

Pop, Ion. Gellu Naum: Poezia contra literaturii. Cluj: Casa Cărții de Știință, 2001.

Popescu, Simona. Clava: Critificțiune cu Gellu Naum. Pitești: Paralela 45, 2004. Odiseu.

Spiridon, Vasile. Gellu Naum: Monografie, antologie comentată, receptare critică. Brașov: Aula, 2005. Colecția "Canon."

Vintilă, Isabel. Gellu Naum: O călătorie spre arhetipul interiorităţiii. Iaşi: Timpul, 2010. 


\section{Sesto Pals}

Born Simion Şestopal, 1912, Odessa; died 2012, Tel Aviv.

\section{PRIMARY}

Omul ciudat. Bucharest: Vinea, 1998. Rev. ed.: Bucharest: Paideia, 2003.

Scrieri în proză 1950-1970. Ed. Michael Finkenthal. Bucharest: Tracus Arte, 2014.

\section{SECONDARY}

Finkenthal, Michael. Sesto Pals, poetul prăpastiei: O viaţă in secol. Bucharest: Tracus Arte, 2014.

\section{Saşa Pană}

Born Alexandru Binder, 1902, Bucharest; died 1981, Bucharest.

\section{PRIMARY}

Răbojul unui muritor: Strofe banale. Bucharest: Lupta, 1926.

Diagrame. Bucharest: Unu, 1930. Rpt. with Viața romanțată a lui Dumnezeu (2006); rpt. with Călătorie cu funicularul (2012).

Echinox arbitrar. Bucharest: Unu, 1931. Rpt. with Viața romanțată a lui Dumnezeu (2006).

Viața romanțată a lui Dumnezeu. Bucharest: Unu, 1932. Rpt. 2006 with 2 other works (see below); rpt. with Călătorie cu funicularul (2012).

Cuvântul talisman. Bucharest: Unu, 1933. Rpt. with Călătorie cu funicularul (2012).

Călătorie cu funicularul: Poeme. Bucharest: Unu, 1934. Rpt. 2012 with 5 other works (see below).

Sadismul adevărului. Bucharest: Unu, 1936. Rpt.: Ed. Ion Pop. Cluj-Napoca: Dacia, 2009. Colecţia Avangarda.

Iarba fiarelor. Bucharest: Unu, 1937. Rpt. with Călătorie cu funicularul (2012).

Vladimir. Bucharest: Unu, 1938.

Munții noaptea neliniștea. Bucharest: Unu, 1940. Rpt. with Călătorie cu funicularul (2012).

Pentru libertate: Poeme. Bucharest: Colectia Orizont, 1945.

Plecări fără ancoră. Bucharest: Steaua Artei, 1946.

Tîlbîc, Tureatcă E Co. Bucharest: Socec, 1948.

A fost odată - şi nu va mai fi! Bucharest: Ed. de Stat, 1949.

Poeme și poezii: Alese din cărţi şi din sertar (1925-1965). Bucharest: Ed. Pentru Literatură, 1966.

Antologia literaturii române de avangardă. Bucharest: Ed. Pentru Literatură, 1969. (Ed. Pană.)

Prozopoeme. Bucharest: Minerva, 1971.

Născut în '02: Memorii - file de jurnal - evocări. Bucharest: Minerva, 1973.

Culoarea timpului: Poeme. Bucharest: Cartea Românească, 1977. 
Viața romanțată a lui Dumnezeu. Ed. Ion Pop. Cluj-Napoca: Dacia, 2006. Colecţia Avangarda. (Collects 3 books originally pub. by Editura Unu, 1930-1932.) Sadismul adevărului. Ed. Ion Pop. Cluj-Napoca: Dacia, 2009. Colecţia Avangarda. Călătorie cu funicularul. Ed. Nicolae Tzone. Bucharest: Vinea, 2012. Avangarda. (Collects 6 books originally pub. by Editura Unu, 1930-1940.)

Prezentări. Ed. Vladimir Pană and Ion Pop. Bucharest: Tracus Arte, 2012.

\section{Paul Păun}

Born Zaharia Herşcovici, 1915, Bucharest; died 1994, Haifa. Changed name to Zaharia Zaharia; published under the names Paul Păun, Paul Paon, and Paul Paon Zaharia. Also known as "Yvenez."

\section{PRIMARY}

Plămânul sălbatec. Bucharest: "Bucovina"/I.E. Torouțiu, 1939.

Marea palidă. Bucharest: n.p., 1945. Colecția suprarealistă.

Brevet lovaj. Bucharest: n.p., 1945. Colecția suprarealistă. Suppl. to Teodorescu, Butelia de Leyda. Facsim. rpt. in Yaari, ed., "Infra-noir" un et multiple 285.

Les Esprits animaux. Bucharest: Imp. Socec, 1947. Infra-noir. Facsim. rpt. in Infranoir rpt. (1996) and in Yaari, ed., "Infra-noir" un et multiple 318-25.

La Conspiration $d u$ silence. Bucharest: Imp. Socec, 1947. Infra-noir. Facsim. rpt. in Infra-noir rpt. (1996) and in Yaari, ed., "Infra-noir" un et multiple 326-33.

La Rose parallèle. Haifa: n.p. 1975. (As Paul Paon Zaharia.) Rpt. forthcoming: Cordes-sur-Ciel: Éditions Rafael de Surtis.

\section{SECONDARY}

Ierunca, Virgil. "Paul Păun sau despre discursul antimetodei." Subiect şi predicat. Bucharest: Humanitas, 1993. 224-33.

Magliocco, Giovanni. “La 'Semence incendiaire' d'une parole nouvelle: Plămânul sălbatec de Paul Păun." Philologica Jassyensia 19 (2014): 83-95.

Yaari, Monique. "Dossier Paul Paon: Paul Paon entre deux langues." Pleine Marge 36 (Dec. 2002): 61-84.

—. "Paul Paon ou le 'hurle-silence.'” Yaari, Monique, ed. "Infra-noir," un et multiple 151-98.

—. “Paul Paon's Sur-surreal Chimera." Utopian Studies 5.1 (1994): 108-19.

\section{EXHIBITION CATALOGUES}

London Gallery. Three Types of Automatism: Works by Ernst Martin, Paul Păun, Scottie Wilson. London: London Gallery, 1948. 18 Feb.-20 March 1948.

\section{Jules Perahim}

Born Jules Blumenfeld, 1914, Bucharest; died 2008, Paris. Also signed "S. Perahim." 


\section{SECONDARY}

Centenar Perahim. Spec. issue of Caietele avangardei 3 (2014).

Perahim. Text, Édouard Jaguer. Paris: Non lieu, 1978.

Perahim. Text, Édouard Jaguer. Paris: Arcane 17, 1990.

\section{EXHIBITION CATALOGUES}

Galerie André François Petit. Perahim. Pref. Alain Jouffroy. Paris: Galerie André François Petit, 1971. 15 June-9 July 1971.

Galerie Mony Calatchi. Perahim. Pref. Édouard Jaguer. Paris: Galerie Mony Calatchi, 1973. 1973.

Galerie Mony Calatchi. Perahim: L'Alphabet: Album de vingt-deux lithographies. Pref. Marina Vanci. Paris: Galerie Mony Calatchi, 1975. 1975.

Galerie d'Art A.C.A.P. Perahim. Text Gilles Plazy. Le Touquet: Galerie d'Art A.C.A.P., 1987.

Musée d'art moderne et contemporain de Strasbourg. Perahim: La Parade sauvage. Strasbourg: Musées de la ville de Strasbourg, 2014. 14 Nov. 2014-1 March 2015.

\section{Claude Sernet}

Born Ernest Spirt, 1902, Târgu Ocna, Romania; died 1968 in Paris. First texts published under the pseud. "Michael Cosma."

\section{PRIMARY}

Cinéma 33. Spec. issue of Cahiers jaunes 4 (1933). (Ed. Sernet.)

Commémorations. Paris: Tschann, 1937.

Un Jour et une nuit. Paris: Sagesse, 1938.

Mais une île peut-être, un rivage-. Paris: n.p., 1948.

Enfin, ces neufs poèmes. N.p.: n.p., 1949.

Les Pas recomptés. Paris: Seghers, 1962.

\section{SECONDARY}

Gourdet, Michel. Claude Sernet. Rodez: Subervie, 1981. Visages de ce temps 30. . Claude Sernet. Paris: Oxus, 2005. Les Étrangers de Paris: Les Roumains de Paris.

\section{Virgil Teodorescu}

Born 1909, Cobadin, Romania; died 1987, Bucharest. Signed his works in French "Virgil Teodorescou."

\section{PRIMARY}

Blănurile oceanelor. Bucharest: Tiparul Universitar, 1945. Colecția suprarealistă.

Butelia de Leyda. With a "Brevet de lovaj" and four lovaje by Paul Păun. Bucharest: Tipografia “Bucovina," 1945. Colecția Suprarealistă. Rpt. in Blănurile oceanelor (1969) 69-106. 
Au lobe du sel. Bucharest: Imp. Socec, 1947. Infra-noir. Facsim. rpt. in Infra-noir rpt. (1996) and in Yaari, ed., "Infra-noir" un et multiple 350-57.

La Provocation. Bucharest: Imp. Socec, 1947. Infra-noir. Facsim. rpt. in Infra-noir rpt. (1996) and in Yaari, ed., "Infra-noir" un et multiple 358-65.

Blănurile oceanelor. Bucharest: Ed. Pentru Literatură, 1969.

Un ocean devorat de licheni urmat de Poemul regăsit: Cu şase stilamancii. Bucharest: Cartea românească, 1984.

\section{SECONDARY}

Vlaicu, Claudia Mihaela. Virgil Teodorescu: Monografia operei. Piteşti: Tiparg, 2013.

\section{Trost}

Born Dolfi Trost, 1916, Brăila, Romania; died 1966, purportedly in Chicago. Signed his mature works "Trost" and his early essays in the press mostly "D. Trost."

PRIMARY

Vision dans le cristal: Oniromancie obsessionnelle et neuf graphomanies entoptiques. Bucharest: Éd. de l'Oubli, [1945].

Le Profil navigable: Négation concrète de la peinture. Bucharest: Éd. de l'Oubli, 1945.

La Connaissance des temps. Bucharest: Imp. Independenta, 1946. Surréalisme.

Le Même du même. Bucharest: Imp. Socec, 1947. Infra-noir. Facsim. rpt. in Infra-noir rpt. (1996) and in Yaari, ed., "Infra-noir" un et multiple 334-41.

Le Plaisir de flotter. Bucharest: Imp. Socec, 1947. Infra-noir. Facsim. rpt. in Infra-noir rpt. (1996) and in Yaari, ed., "Infra-noir" un et multiple 342-49.

Visible et invisible. Paris: Arcanes, 1953.

Librement mécanique. N.p.: n.p., 1955. (Paris: En dépot au "Minotaure.")

SECONDARY

Finkenthal. Michael. D. Trost: Intre realitatea visului şi visul ca realitate. Bucharest: Tracus Arte, 2013.

Nicol, Françoise. "Trost ou le 'plaisir de flotter.'” Yaari, Monique, ed. "Infra-noir," un et multiple 101-50.

Militaru, Petrişor. Suprarealismul lui D. Trost. Craiova: Ed. Universitaria, 2014.

\section{Tristan Tzara}

Born Samuel Rosenstock, 1896, Moinești, Romania; died 1963, Paris. Also briefly used pseud. S. Samyro.

PRIMARY

La Première Aventure céléste de Mr. Antipyrine. Zurich: n.p., 1916. Collection Dada. Trans. as The First Celestial Adventure of Mister Benzedrine. Trans. Elmer Petersen. Colorado Springs: Press at Colorado College, 1996. 
Vingt-cinq poèmes. Zurich: n.p., 1918. Collection Dada. Expanded ed.: Vingt-cinq-etun poèmes. Paris: Fontaine, 1946. Collection "L'Âge d'or."

Cinéma calendrier du cœur abstrait; Maisons. Paris: Au Sans Pareil, 1920. Collection

Dada. Facsim. rpt. (with trans. by Mary Ann Caws as Cinema Calendar of the Abstract Heart; Houses): Ann Arbor: Thomas P, 1982.

Sept manifestes Dada. Paris: Budry, 1924. Editions du diorama. Expanded ed.:

Lampisteries; précédées des Sept manifestes Dada. Paris: Pauvert, 1963. Trans. as

Seven Dada Manifestos and Lampisteries. Trans. Barbara Wright. London: Calder, 1977.

Mouchoir de nuages: Tragédie en 15 actes. Paris: Galerie Simon, 1925.

Indicateur des chemins de coeur. Paris: Bucher, 1928.

De nos oiseaux: Poèmes. Paris: Kra, 1929.

L'Arbre des voyageurs. Paris: Éd. De la Montagne, 1930.

L'Homme approximatif. Paris: Fourcade, 1931. Trans. as Approximate Man and Other

Writings. Trans. Mary Ann Caws. Detroit: Wayne State UP, 1973; rpt. as Approximate Man \& Other Writings. Boston: Black Widow P, 2005.

Où boivent les loups. Paris: Cahiers Libres, 1932.

L'Antitête. Paris: Cahiers libres, 1933.

Primele poeme ale lui Tristan Tzara. Ed. Sașa Pană. Bucharest: Unu, 1934. Facsim. of MSS: Litanii avantdada. Ed. Alexandru Condeescu. Bucharest: Muzeul Literaturii Române, 1996. Trans. as Primele Poeme/First Poems. Trans. Michael Impey and Brian Swann. Berkeley: New Rivers P, 1976. French trans. as Les Premiers poèmes. Trans. Claude Sernet. Paris: Seghers, 1965.

Grains et issues. Paris: Denoël et Steele, 1935.

La Deuxième Aventure céleste de M. Antipyrine. Paris: Réverbères, 1938.

Midis gagnés: Poèmes. Paris: Denoël, 1939. Expanded ed.: Paris: Denoël, 1948.

Le Cour à gaz. Paris: G.L.M., 1946. Trans. as The Gas Heart: The Dada Masterpiece of

Drama. Trans. Eric v.d. Luft. North Syracuse: Gegensatz Press, 2008.

Entre-temps. Paris: Point du jour, 1946. Le Calligraphe 4.

Le Signe de vie. Paris: Bordas, 1946.

Terre sur terre. Geneva: Trois Collines, 1946.

La Fuite: Poème dramatique en quatre actes et un épilogue. Paris: Gallimard, 1947.

Morceaux choisis. Paris: Bordas, 1947.

Le Surréalisme et l'après-guerre. Paris: Nagel, 1947. Littérature.

De mémoire d'homme. Paris: Bordas, 1950.

Parler seul: Poème. Paris: Maeght, 1950.

La Face intérieure.Paris: Seghers, 1953.

13 Poems. Trans. Franklin Rosemont. Chicago: Black Swan P, 1969.

CEuvres complètes. Ed. Henri Béhar. 6 vols. Paris: Flammarion, 1975-1991.

Zürich, Dadaco, Dadaglobe: The Correspondence between Richard Huelsenbeck, Tristan

Tzara and Kurt Wolff (1916-1924). Ed. Richard Sheppard. Tayport, Fife, Scotland: Hutton P, 1982. 
Dada terminus: Tristan Tzara-E.L.T. Mesens, correspondance choisie, 1923-1926. Ed. Stéphane Massonet. Brussels: Devillez, 1997.

Chanson Dada: Selected Poems. Trans. Lee Harwood. Boston: Black Widow P, 2005.

\section{SECONDARY}

Béhar, Henri, ed. Chassé-croisé Tzara-Breton: Actes du colloque international ParisSorbonne 23-25 mai 1996. Lausanne: L'Âge d'homme, 1997. Cahiers du Centre de recherche sur le surréalisme.

Béhar, Henri. Tristan Tzara. Paris: Oxus, 2005.

Blachère, Jean-Claude. Le Modèle nègre: Aspects littéraires du mythe primitiviste au XXe siècle chez Apollinaire, Cendrars, Tzara. Dakar: Nouvelles Éd. africaines, 1981.

Browning, Gordon Frederick. Tristan Tzara: The Genesis of the Dada Poem, or, From Dada to Aa. Stuttgart: Heinz, 1979. Stuttgarter Arbeiten zur Germanistik 56.

Buot, François. Tristan Tzara: L'Homme qui inventa la révolution Dada. Paris: Grasset, 2002.

Les Cahiers Tristan Tzara. Moineşti: Association culturelle et littéraire 'Tristan Tzara,' 1998- .

Codrescu, Andrei. The Posthuman Dada Guide: Tzara and Lenin Play Chess. Princeton: Princeton UP, 2009.

Dachy, Marc. Il y a des journalistes partout: De quelques coupures de presse relatives à Tristan Tzara et Andre Bréton. Paris: Gallimard, 2015. L'Infini.

—. Tristan Tzara: Dompteur des acrobates: Dada Zurich. Paris: Échoppe, 1992.

Fauchereau, Serge. "Tristan Tzara et l'avant-garde roumaine." Critique 300 (May 1972): 416-29.

Forcer, Stephen. "Before They Were Famous: Tristan Tzara, Nationhood, and Poetry." Paris-Bucharest, Bucharest-Paris: Francophone Writers from Romania. Ed. Anne Quinney. Amsterdam: Rodopi, 2012. Faux Titre 367. 71-94.

- Modernist Song: The Poetry of Tristan Tzara. Leeds: Legenda, 2006.

Girault, Jacques, and Bernard Lecherbonnier, eds. Tristan Tzara, le surréalisme et l'internationale poétique. Paris: Harmattan, 2000. Itinéraires \& contacts de cultures 29.

Harwood, Lee. Tristan Tzara: A Bibliography. London: Aloes Books, 1974.

Hentea, Marius. TaTa Dada: The Real Life and Celestial Adventures of Tristan Tzara. Cambridge: MIT P, 2014.

Ko, Won. Buddhist Elements in Dada: A Comparison of Tristan Tzara, Takahashi Shinkichi, and Their Fellow Poets. New York: New York UP, 1977. New York University Studies in Comparative Literature 8.

Lista, Giovanni. "Marinetti et Tzara." Les Lettres nouvelles May-June 1972: 82-97.

Macarie, Victor. “Tristan Tzara: Evocări şi documente." Manuscriptum 21.3-4 (1990): 97-103. 
Papachristos, Katherine. L'Inscription de l'oral et de l'écrit dans le théâtre de Tristan Tzara. New York: P. Lang, 1999. Currents in Comparative Romance Languages and Literatures 69.

Peterson, Elmer. Tristan Tzara: Dada and Surrational Theorist. New Brunswick: Rutgers UP, 1971.

Tison-Braun, Micheline. Tristan Tzara, inventeur de l'homme nouveau. Paris: Nizet, 1977.

\section{Urmuz}

Born Demetru Demetrescu-Buzău, 1883, Curtea de Argeș, Romania; died 1923, Bucharest

\section{PRIMARY}

Algazy şi Grummer. Ed. Saşa Pană. Bucharest: Colecția Editurii Unu, 1930. Rpt. as Pagini bizare. Bucharest: Minerva, 1970. New ed. based on Academia Română MS 4486: Schiţe şi nuvele aproape - futuriste. Ed. Ion Pop. Bucharest: Tracus Arte, 2012. Biblioteca avangardei. Bilingual ed.: Pagini bizare (Weird pages). Trans. Stavros Deligiorgis. Bucharest: Cartea Românească, 1985. Trans.: The Complete Works of Urmuz. Trans. Miron and Carola Grindea. London: Atlas, 2006.

\section{SECONDARY}

Balotă, Nicolae. Urmuz. Cluj: Dacia, 1970.

Cubleşan, Constantin. Urmuz în conştiinţa criticii. Bucharest: Cartea Românească, 2014.

Segărceanu, Amalia. UrMUZA cu BOCANCI: Un studiu asupra scrierilor lui Urmuz. Bucharest: Vinea, 2004.

\section{Ion Vinea}

Born Ion Eugen Iovanaki, 1895, Giurgiu, Romania; died 1964, Bucharest.

PRIMARY

Descântecul și Flori de lampă. Bucharest: Biblioteca Dimineața, 1925.

Paradisul suspinelor. Bucharest: Cultura națională, 1930. Biblioteca portativă. Facsim. rpt.: Bucharest: ICARE, 2006.

Ora fintînilor: Poezii. Bucharest: Ed. Pentru Literatura, 1964.

Lunatecii. Bucharest: Ed. Pentru Literatură, 1965. 2 vols.

Venin de mai: Roman. Cluj: Dacia, 1971.

Opere. Ed. Elena Zaharia Filipaș. Bucharest: Minerva, 1984- . 8 vols. to date.

SECONDARY

Bucerzan, Dana. Ion Vinea: O abordare semantic-textuală a creației poetice. ClujNapoca: Dacia, 2001. Discobolul.

Mioc, Simion. Opera lui Ion Vinea. Bucharest: Minerva, 1972. Universitas. 
Pachia Tatomirescu, Ion. Întâiul dadaism (Le Premier Dadaïsme) (The First Dadaism). Timișoara: Aethicus, 2009. Biblioteca absolventului.

Podosu, Ion. Ion Vinea, un melancolic al avangardei românești: Eseu critic. ClujNapoca: Limes, 2010. Colecția Monografii.

Sălăgean, Sergiu. Ion Vinea. Bucharest: Eminescu, 1971. Biblioteca critică 49.

Zaharia, Elena. Ion Vinea. Bucharest: Cartea românească, 1972.

\section{Ilarie Voronca}

Born Eduard Marcus, 1903, Brăila, Romania; died 1946, Paris.

\section{PRIMARY}

Restrişti. Bucharest: Rahova, 1923. Rpt. in Zodiac (1992).

Colomba. Paris: Imp. Union, 1927. Facsim. rpt.: Bucharest: ICARE, 2001. Rpt. in Zodiac (1992).

Ulise. Paris: Imp. Union, 1928. Colecțiunea Integral. Facsim. rpt.: Bucharest: ICARE, 2001. Rpt. as Ulise; Brățara nopților. Cluj-Napoca: Dacia, 2003. French trans as Ulysse dans la cité. Trans. Roger Vailland. Paris: Sagittaire, 1933. Rpt. Paris: Temps des cerises, 1999.

Brățara nopților. Bucharest: Unu, 1929. Rpt. with Ulise (2003).

Plante şi animale; Terase. Paris: Imp. Union, 1929. Colecţia Integral. Facsim. rpt.:

Bucharest: ICARE, 2001. Rpt. in Zodiac (1992).

A doua lumină. Bucharest: Unu, 1930. Rpt. in Act de prezență (Cluj: Dacia, 1972 ) and

A doua lumină (Bucharest: Minerva, 1996).

Invitație la bal: 1924-1925. Bucharest: Unu, 1931.

Petre Schlemihl. Bucharest: Tipografia "Bucovina", 1932. French trans. by the author as Poèmes parmi les hommes. Paris: Cahiers du Journal des poètes, 1934.

Act de prezență. Bucharest: Cartea cu semne, 1932. Rpt. in Act de prezență (Cluj:

Dacia, 1972 ) and A doua lumină (Bucharest: Minerva, 1996).

Patmos și alte șase poeme. Bucharest: Vremea, 1933.

Patmos. Paris: Cahiers libres, 1934. Rpt.: La Bastide d'Orniols; Pont de l'épée, 1977. Permis de séjour: Poèmes. Paris: Corrêa, 1935.

La Poésie commune. Paris: G.L.M., 1936. Rpt.: Paris : Plasma, 1979.

La Joie est pour l'homme: Poèmes, suivis de 3 poèmes à la gloire du sommeil. Marseille:

Cahiers du sud, 1936.

Pater Noster, suivi de Ébauches d'un poème. Verviers: Avant-Poste, 1937 (limited ed.).

Paris: Corrêa, 1937 (trade ed.).

Amitié des choses. Paris: Sagesse, 1937.

L'Apprenti Fantôme et Cinq Poèmes de septembre. Paris: Librairie les lettres; Presses du Hibou, 1938. Rpt. (L'Apprenti Fantôme only): Brussels: L'Arbre, 1992.

Le Marchand de quatre saisons. Brussels: Cahiers du Journal des Poètes, 1938.

Beauté de ce monde. Paris: Sagittaire, 1940.

Lord Duveen, ou, L'invisible à la portée de tous. Rodez: Ilot, 1941. Rpt.: Geneva: Vernay, 1977. 
La Confession d'une âme fausse. Rodez: Méridien, 1942.

Oisiveté. Paris: Sagesse, 1943. Les Feuillets de "Sagesse" 60.

La Clé des réalités. Paris: Meridien, 1944.

L'Interview. Marseille: Vigneau, 1944.

Henrika: Grand Roman pour les petits, petit roman pour les grands. Paris: n.p., 1945.

Contre solitude: Poèmes. Paris: Bordas, 1946.

Petit Manuel du parfait bonheur (Mic manual de fericire perfectă). With Romanian trans. by Saşa Pană. Bucharest: Cartea românească, 1973.

Zodiac: Poezii. Ed. Ion Pop. Bucharest: Minerva, 1992. (Includes Restrişti, Colomba, Plante şi animale, Brățara nopților, and Zodiac.)

\section{SECONDARY}

Bouloc, Denys-Paul. Ilarie Voronca. Rodez: Subervie, 1961. Visages de ce temps 8. Dauphin, Christophe. Ilarie Voronca: Le Poète intégral. Cordes-sur-Ciel: Rafael de Surtis; Paris: Editinter, 2011.

Grün, Ecaterina. La Route chez Tristan Tzara, Benjamin Fondane et Ilarie Voronca. Cordes-sur-Ciel: Surtis, 2006. Collection "Art, artistes."

Pop, Ion. A scrie şi a fi: Ilarie Voronca şi metamorfozele poeziei. Bucharest: Cartea Românească, 1993. 2nd ed. Bucharest: Cartea Românească, 2007.

\section{Jointly Authored Works}

Gherasim Luca, Paul Păun, Trost, and Virgil Teodorescou. Collection surréaliste Infra-noir. 8 vols. Bucharest: Imp. Socec, 1947. (Includes two works by each of the four authors; see the individual works above.) Facsim. rpt.: Paris: Maison de verre, 1996. Facsim. rpt. in Yaari, ed., "Infra-noir" un et multiple 302-65.

Gherasim Luca, Gellu Naum, Paul Păun, Virgil Teodorescou, and Trost. Éloge de Malombra: Cerne de l'amour absolu. Bucharest: Socec, 1947. S Surréalisme. Facsim. rpt. Yaari, Monique, ed., "Infra-Noir" 366-72.

- L'Infra-noir: Préliminaires à une intervention sur-thaumaturgique dans la conquête du desirable. Bucharest: n.p., 1946. S Surréalisme. Facsim. rpt. in Infranoir rpt. (1996) and in Yaari, Monique, ed., "Infra-Noir" 289-301.

. "Le Sable nocturne." Le Surréalisme en 1947. Ed. André Breton and Marcel Duchamp. Paris: Maeght, 1947. 56-58. Facsim. rpt. Yaari, Monique, ed., "InfraNoir" 373-75.

Gherasim Luca and Trost. Dialectique de la dialectique: Message adressé au mouvement surréaliste internationale. Bucharest: Imp. Slova, 1945. S Surréalisme. Facsim. rpt.: Bucharest: ICARE, 2001. Facsim. rpt. in Yaari, ed., "Infra-noir" un et multiple 239-69. Trans. as "Dialectics of the Dialectic." Surrealism Against the Current: Tracts and Declarations. Ed. Michael Richardson and Krzysztof Fijalkowski. London: Pluto, 2001. 32-41.

- Présentation de graphies colorées, de cubomanies et d'objets.Bucharest: Imp. Independența, 1945. Yaari, Monique, ed., "Infra-Noir" 270-84. 
Naum, Gellu, and Virgil Teodorescu. Spectrul longevității: 122 de cadavre. Bucharest: Tip. Bucovina, 1946. Colecția suprarealistă.

Naum, Gellu, Paul Păun, and Virgil Teodorescou. Critica mizeriei. Bucharest: Ed. Modernă, 1945.

Copyright (C) 2015 Timothy Shipe 